\title{
Biosensors for bacterial detection
}

\begin{abstract}
In the past decades, biosensors have been developed for detection and diagnose of the presence of bacteria in various service environments. Biological recognition elements, transducer and signal amplifier are the main components of a biosensor, affecting the selectivity and sensitivity and signal-responding time of the tool. Various biological materials, including enzymes, antibodies, DNA and aptamers, are combined with the signal transducers made of electrochemical, optical, piezoelectric and thermal detection platforms, as well as nanomaterials, i.e., carbon nanotubes, graphene, metallic nanoparticles and magnetic nanoparticles, to improve the performance of the biosensors. This paper reviews the trends in bacteria detection, the primary biosensor techniques.
\end{abstract}

Volume 2 Issue 6 - 2017

\author{
Shiqiang Chen, Y Frank Cheng \\ Department of Mechanical \& Manufacturing Engineering, \\ University of Calgary, Canada
}

Correspondence: Frank Cheng Y, Department of Mechanical \& Manufacturing Engineering, University of Calgary, Calgary, Alberta,T2N IN4, Canada, Tel (+I)403 220 3693, Email fcheng@ucalgary.ca

Received: June 27, 2017 | Published: July 14, 2017

Keywords: biosensors, bacteria, biological recognition elements, transducers, signal amplifiers

\section{Introduction}

Bacteria are present widely in various environments, such as soils, marine and estuarine water, intestinal tract of animals, and waste water. Normally, the bacteria are not visible to human eyes due to their small size of usually a few micrometers in length, with the shapes of spheres, rods or spirals. The presence of bacteria can be harmful to human/animal health, food safety, environmental conservation, and infrastructure integrity. Therefore, development of accurate, effective and rapid methods for bacterial detection is essential to prevention and control of harmful of bacteria in the environments. ${ }^{1}$ During the past century, extensive methods have been developed for bacterial detection, including traditional culturing methods, immunological techniques, molecular biological techniques and biosensors. Particularly, biosensors have been one of the most important techniques due to demonstrated excellent performance, i.e., low cost, fast response, high sensitivity, and high selectivity. ${ }^{2}$

The principle of a biosensor is to use biological materials (i.e., antimicrobial peptides, lectin, antibody and aptamer) to recognize a target molecule, and produce detectable signals. The components of the biosensor include biological sensing element, transducer, signal amplifier and signal processer. When the biological component recognizes the analyte to generate a catalytic or binding event, the detectable signals, such as electrical signals or optical signals, are produced and captured by a transducer. The signals are proportional to the analyte concentration. The performance of the biosensors is featured by their response time, dynamic range, limit of detection, single-to-noise ratio, and specificity. ${ }^{3}$ These parameters are strongly related to the biological sensing element, transducer and signal amplifier, i.e., the most significant components for a biosensor.

\section{Discussion}

\section{Biological sensing elements}

The most important component for a biosensor is the biological recognition element, which determines the selectivity of the biosensors. The biological recognition element is closely related to the analytic targets, i.e., bacterial components and the whole bacteria, in development of bacterial biosensors. The target bacterial components, i.e., DNA, RNA (e.g., rRNA), intracellular proteins such as enzymes and secreted substances, can be liberated by disrupting or lysing bacteria. As a result, molecular recognition elements and cell recognition elements have been developed as the most common recognition elements in bacterial detection. Similar to other biosensors, the molecular recognition elements in bacterial biosensors include receptors, enzymes, antibodies, nucleic acids, molecular imprints and lectins. Based on the nature of the molecular recognition, the biosensor can base biocatalysis or affinity mechanism to recognize the bacterial components. Enzymes are the typical biological catalysts. While the oldest ones, they are still commonly used as the biorecognition element in detecting many different analytes, which range in size from individual ions and small molecules to nucleic acid and proteins. ${ }^{4}$ Enzymes are characterized with high turnover rates and a high selectivity for a desired analyte. These properties, together with the simplicity of the enzymatic biorecognition and the relatively low cost, make enzymatic biocatalytic systems almost indispensable for detecting bacterial components. Affinity bacterial biosensors rely on a selective binding interaction between the bacterial components and the biological recognition element such as antibody, nucleic acid, aptamers and phages. High affinity antibodies and DNA are important to development of affinity bacterial biosensors. Aptamers and phages are powerful tools that excel over antibodies in sensibility, stability, multi-detection, in vivo measurements and regeneration. Phages are superior in stability, screening of affinity-based target bacterial molecules, and easy production. ${ }^{5}$ Living cells have also been used as recognition elements in bacterial biosensors. ${ }^{6}$ The analytical signal is detected by measuring the general metabolic status of such living cells. Generally whole cells are advantageous over enzymes due to high stability, reduced purification requirements, low cost of preparation, and effective cofactor regeneration. ${ }^{7}$

\section{Transducers}

When the biological recognition element reacts with a target analyte, detectable signal is produced through a transducer, which is an analytical tool to provide output quantity proportional to the input quantity. Based on the detectable signals, i.e., electric current, potential, impedance, fluorescence, piezoelectricity and temperature, the transduction for bacterial biosensors can be categorized into four main classes, i.e., electrochemical, optical, piezoelectric (mass detection methods) and thermal detection platforms. Electrochemical 
biosensors for bacterial detection, such as amperometric, potentiometric and impedance biosensors, are the most widespread class as they are economic and have a fast response time. The transduces of these biosensors are usually made of inert materials, such as glassy carbon, gold, platinum, or graphite. Compared to amperometric and potentiometric biosensors, impedimetric biosensors are a very promising choice for the detection of whole bacteria, because they are label free, less costly, highly sensitive, and not affected by the presence of other analytes or colored compounds in the sample matrix. ${ }^{8}$ Optical biosensors are rapid, specific, sensitive, cost effective and suitable for real-time on-site bacterial detection. They are often divided into two categories, i.e., fluorescence based and label free. The tunable fluorescence properties of semiconductor nanoparticles, e.g., zinc oxide nanomaterials, titanium dioxide nanomaterials and quantum dots, have been used for the photonic detection of biorecognition processes. The major disadvantage of using fluorescence-based optical biosensors for bacterial detection is the requirement for sample labeling with fluorescent reagents, which adds time and cost to the procedure. Surface plasmon resonance (SPR) is a label-free method of optical sensing, and it has been employed for the detection of whole bacterial cells using a variety of bioreceptors, including antibodies, bacteriophages, and lectins. ${ }^{9}$ Piezoelectric biosensors are based on the coupling of the bioelement with a piezoelectric component, usually a quartz-crystal coated with gold electrodes. Many types of materials (i.e., quartz, tourmaline, lithium niobate or tantalate, oriented zinc oxide, and aluminum nitride) exhibiting the piezoelectric effect are used as the transducer. For example, quartz-crystal microbalance (QCM) sensors are label-free piezoelectric biosensors, and they have been developed for the detection of whole bacterial cells, including Escherichia coli, Salmonella enterica serovar Typhimurium, Campylobacter jejuni and Bacillus anthracis. ${ }^{10}$ Temperature sensors is the transducer for a thermal biosensor, and which are usually thermistors and thermocouples. Their high sensitivity to thermal changes makes thermistors ideal for such applications. Unlike other transducers, thermal biosensors do not need frequent recalibration and are insensitive to the optical and electrochemical properties of the sample. Common applications of this type of biosensor include the detection of pesticides and pathogenic bacteria. ${ }^{11}$

\section{Signal amplifiers}

Similar to the other biosensors, signal amplification is the direct method for improving the sensitivity of the biosensors for bacterial detection. Nanomaterials have been widely used as the signal amplifiers in development of bacterial biosensors due to their good conductivity, good photoelectrochemical activity, and large surface/ volume ratio. Three approaches have been developed for signal amplification of nanomaterial biosensors for bacterial detection. ${ }^{12}$ Metal and semiconductor nanoparticles with enzyme-like activities are directly used as active labels for the electrochemical amplification detection of bacterial proteins. For example, $\mathrm{Fe}_{3} \mathrm{O}_{4}$ nanoparticles and $\mathrm{Fe}_{3} \mathrm{O}_{4}$ nanoparticles-based hybrid materials can exhibit enhanced peroxidase-like activity, and have been used for detecting bacteria. ${ }^{13}$ Nanomaterials are used as carriers to load a large quantity of active species. Enzyme-functionalized nanomaterials are used as the label to enhance the detection sensitivity, which is achieved by increasing the enzyme loading toward a sandwich immunological reaction event in bacterial detection. ${ }^{14}$ The carbon nanotubes, bio-compatible metal/ metal oxide nanomaterials (e.g., $\mathrm{Au}, \mathrm{Ag}$, and $\mathrm{ZnO}$ ), graphene based materials (graphene, graphene oxide and reduced graphene oxide) are some of the promising materials to amplify the signal. Highly attractive electrochemical properties and electrocatalytic activity of these nanomaterials have facilitated achievement of enhanced signal amplification needed for the fabrication of ultrasensitive electrochemical affinity biosensors to detect a lot of analytes, including bacterial components and whole bacteria. ${ }^{15}$ Recent advancement in nanotechnology allows the development of new nanostructured materials, which impacts greatly the overall performances of the bacterial biosensors. ${ }^{16,17}$

\section{Conclusive remarks}

Despite the considerable progress made in the detection of bacteria using biosensors, the sensitivity of the biosensors is required to improve for rapid identification of bacterial species and strains. Additional challenges include the ability to detect bacteria in real environments and the response time. To meet the increasing demand for bacterial detection, innovative biosensor devices featuring with a satisfactory combination of precision, sensitivity, specificity, speed and usability, need to be developed. The interaction mechanism between the recognition element and bacterial cells can provide a great help to develop specific detection strategies. The new functioned materials, i.e., nanoparticles, nanowires and quantum dots, provide access to sensitive signal transduction methods to detect bacterial cells. An excellent integration of biochemistry, biology, nanotechnology, physics and electronics would facilitate the development of highperformance biosensors for bacteria detection.

\section{Acknowledgements}

This work was supported by the University of Calgary's Eyes High Postdoctoral Scholar Program.

\section{Conflict of interest}

The author declares no conflict of interest.

\section{References}

1. Sanvicens N, Pastells C, Pascual N, et al. Nanoparticle-based biosensors for detection of pathogenic bacteria. Trends Anal Chem. 2009;28(11):1243-1252.

2. Liu X, Marrakchi M, Xu D, et al. Biosensors based on modularly designed synthetic peptides for recognition, detection and live/dead differentiation of pathogenic bacteria. Biosens Bioelectron. 2016;80:9-16.

3. Saha K, Agasti SS, Kim C, et al. Gold nanoparticles in chemical and biological sensing. Chem Rev. 2012;112(5):2739-2779.

4. Sekretaryova AN, Eriksson M, Turner AP. Bioelectrocatalytic systems for health applications. Biotechnol Adv. 2016;34(3):177-197.

5. Yue H, He Y, Fan E, et al. Label-free electrochemiluminescent biosensor for rapid and sensitive detection of pseudomonas aeruginosa using phage as highly specific recognition agent. Biosens Bioelectron. 2017;94:429432.

6. Templier V, Roux A, Roupioz Y, et al. Ligands for label-free detection of whole bacteria on biosensors: A review. Trends Anal Chem. 2016;79:7179

7. Venkatesh AG, Sun A, Brickner H, et al. Yeast dual-affinity biobricks: Progress towards renewable whole-cell biosensors. Biosens Bioelectron. 2015;70:462-468.

8. Ahmed A, Rushworth JV, Hirst NA, et al. Biosensors for whole-cell bacterial detection. Clin Microbiol Rev. 2014;27(3):631-646.

9. Nguyen HH, Park J, Kang S, et al. Surface plasmon resonance: a versatile technique for biosensor applications. Sensors. 2015;15(Basel) 5:1048110510 
10. Saad NA, Zaaba SK, Zakaria A, et al. Quartz crystal microbalance for bacteria application review. $2^{\text {nd }}$ International Conference; Malaysia: IEEE; 2014.

11. Mohanty SP, Kougianos E. Biosensors: a tutorial review. IEEE Potentials. 2006;25(2):35-40.

12. Lei J, Ju H. Signal amplification using functional nanomaterials for biosensing. Chem Soc Rev. 2012;41(6):2122-2134

13. Ju H. Signal Amplification for Highly Sensitive Immunosensing. Journal of Analysis and Testing. 2017;1(1):7.

14. Miranda OR, Li X, Garcia-Gonzalez L, et al. Colorimetric bacteria sensing using a supramolecular enzyme-nanoparticle biosensor. $J \mathrm{Am}$ Chem Soc. 2011;133(25):9650-9653.
15. Kumar S, Ahlawat W, Kumar R, et al. Graphene, carbon nanotubes, zinc oxide and gold as elite nanomaterials for fabrication of biosensors for healthcare. Biosens Bioelectron. 2015;70:498-503.

16. Zhao $\mathrm{Y}, \mathrm{Hu} \mathrm{S}$, Wang $\mathrm{H}$, et al. DNA dendrimer-streptavidin nanocomplex: an efficient signal amplifier for construction of biosensing platforms. Anal Chem. 2017;89(12):6907-6914

17. Wang T, Yu Y, Chen D, et al. Plasmonic indicator by naked eyes with multi-responsive polymer brush as signal transducer and amplifier. Nanoscale. 2017;9:1925-1933. 